\title{
Excitation spectrum in a cylindrical Bose-Einstein gas
}

\author{
Tomoya Isoshima*, and Kazushige Machida \\ Department of Physics, Okayama University, Okayama 700, Japan
}

(December 9, 1997)

\begin{abstract}
Whole excitation spectrum is calculated within the Popov approximation of the Bogoliubov theory for a cylindrical symmetric Bose-Einstein gas trapped radially by a harmonic potential. The full dispersion relation and its temperature dependence of the zero sound mode propagating along the axial direction are evaluated in a self-consistent manner. The sound velocity is shown to depend not only on the peak density, but also on the axial area density. Recent sound velocity experiment on $\mathrm{Na}$ atom gas is discussed in light of the present theory.
\end{abstract}

PACS numbers: 03.75.Fi,05.30.Jp,67.40.Db,67.57.Jj

Much attention has been focused on Bose-Einstein condensation (BEC) since its realization in alkaline atomic gases. This macroscopic quantum phenomenon is unique because it occurs in ideal Bose gas, but real interest lies in the fact of how the particle interaction affects on it. Superfluidity which is absent in ideal Bose gas is understood to be sustained by the particle interaction. By flurrious experimental and theoretical studies, various fundamental properties of Bose-Einstein condensation, including the condensation fraction relative to non-condensate, its temperature dependence and few lowest-lying collective modes have been already elucidated quite thoroughly [1]. However, because of the small limited size of trapped atomic gases these collective modes are all localized so far, thus the spectrum was discrete [2].

Rapid development of experimental techniques to trap larger number of atoms leads to a chance to investigate propagating collective modes, such as zero, first and second sound. In particular, the zero sound mode with the continuous spectrum is intrigue because it gives the critical superfluid velocity above which the system returns to be normal gas. In quantum liquid of superfluid ${ }^{4} \mathrm{He}$ where phonon, roton and maxon are known to exist microscopic description for these modes has not been quite successful because ${ }^{4} \mathrm{He}$ atoms are strongly interacting. In contrast, in the present dilute Bose gases there is a good opportunity to thoroughly understand these low-lying excitations in a microscopic level. In that context a recent zero sound measurement by Andrews et al. [3] on a long cigar shaped $\mathrm{Na}$ atomic gas with length typically $500 \mu \mathrm{m}$ and width $10 \mu \mathrm{m}$ provides an interesting testing ground and challenges theoretical investigation.

The speed of this compressional zero sound mode is known to be given by $c=\sqrt{n_{0} g / m}$ with the repulsive interaction constant $g$ in the case of a homogeneous bulk system where the lowest collective mode is exhausted by the zero sound mode [1]. Here $n_{0}$ is the condensate density. It is not obvious at all that this expression by regarding $n_{0}$ as the peak density is readily applicable to the above experiment as done by Andrews et al. [3] because the system trapped by a harmonic potential is finite and everywhere inhomogeneous.

In fact, Zaremba [5] derives a different formula $c=$ $\sqrt{n_{0}(0) g / 2 m}$ for an idealized cylindrical trap by solving a time-dependent Gross-Pitaevskii equation within the Thomas-Fermi approximation (TFA) with $n_{0}(0)$ being the peak condensate density. The extra factor $\sqrt{2}$ in the denominator comes from a simple geometrical reason that the average condensate density is half the peak density in this parabolic shaped condensate. The same result is also obtained by Kavoulakis and Pethick [6]. Zaremba's expression is far off the experimental data. As pointed out by himself a microscopic calculation is needed.

Here we study the whole excitation spectrum, not only low-lying excitations, but also high energy ones for a cylindrical BEC system which is confined radially by a harmonic potential and infinite axially, approximating the above long cigar shaped trapped gas. We give special attention on zero sound velocity. The present set of the microscopic results, including condensate and noncondensate spatial profile, the relative fraction, their $T$ dependences and the whole excitation spectrum, within Popov approximation of the Bogoliubov theory enables us to draw a self-consistent picture of BEC systems. This theoretical framework has been quite successfully applied to the present dilute atomic BEC systems so far [1].

We start out with the following Hamiltonian in which Bose particles interact with a two-body potential $g \delta\left(\mathbf{r}_{1}-\right.$ $\mathbf{r}_{2}$ ) where $g$ is a positive (repulsive) constant proportional to the s-wave scattering length $a$, namely $g=4 \pi \hbar^{2} a / m$ :

$$
\hat{\mathrm{H}}=\int d \mathbf{r} \hat{\Psi}^{\dagger} h(\mathbf{r}) \hat{\Psi}+\frac{g}{2} \int d \mathbf{r} \hat{\Psi}^{\dagger} \hat{\Psi}^{\dagger} \hat{\Psi} \hat{\Psi}
$$

where the one-body Hamiltonian $h(\mathbf{r})=-\frac{\hbar^{2} \nabla^{2}}{2 m}+V(\mathbf{r})-$ $\mu$, the chemical potential $\mu$ is introduced to fix the total particle number and $V(\mathbf{r})$ is the confined potential. In order to describe the Bose condensation, we assume that the field operator $\hat{\Psi}$ is decomposed into $\hat{\Psi}=\hat{\psi}+\phi$ where the ground state average is given by $\langle\hat{\Psi}\rangle=\phi$. A c-number $\phi$ corresponds to the condensate wave function and $\hat{\psi}$ is a q-number describing the non-condensate. Thus the condensate density is given by $n_{0}=|\phi|^{2}$. The condition that the first order term in $\hat{\psi}$ vanish yields 


$$
h(\mathbf{r}) \phi+g|\phi|^{2} \phi+2 g \rho \phi=0 .
$$

When the non-condensate density $\rho$ is made to zero, it reduces to the Gross-Pitaevskii (GP) equation: $h(\mathbf{r}) \phi+$ $g|\phi|^{2} \phi=0$. The condition that the Hamiltonian be diagonalized gives rise to the following set of eigenvalue equations for $u_{q}$ and $v_{q}$ with the eigenvalue $\varepsilon_{q}$ :

$$
\begin{gathered}
\left\{h(\mathbf{r})+2 g \rho+2 g|\phi|^{2}\right\} u_{q}-g \phi^{2} v_{q}=\varepsilon_{q} u_{q} \\
\left\{h(\mathbf{r})+2 g \rho+2 g|\phi|^{2}\right\} v_{q}-g \phi^{* 2} u_{q}=-\varepsilon_{q} v_{q} .
\end{gathered}
$$

The variational parameter $\rho$ is determined selfconsistently by $\rho=\left\langle\hat{\psi}^{\dagger} \hat{\psi}\right\rangle=\sum_{q}\left(u_{q}^{*} u_{q}+v_{q}^{*} v_{q}\right) f\left(\varepsilon_{q}\right)+$ $\sum_{q} v_{q}^{*} v_{q}$ where $f(\varepsilon)$ is the Bose distribution function. These constitute a complete set of the equations within the Popov approximation. The total particle number density is given as $n_{t}=n_{0}+\rho$.

We now consider a cylindrically symmetric system in the cylindrical coordinate: $\mathbf{r}=(r, \theta, z)$. The system is trapped by a harmonic potential $V(r)=\frac{1}{2} m \omega_{0} r^{2}$ radially and periodic along the $z$-axis whose period is $L$. The eigenfunctions of $u_{q}$ and $v_{q}$ are written as $u_{q}=u_{q}(r) e^{i l \theta} e^{i k z}$ and $v_{q}=v_{q}(r) e^{i l \theta} e^{i k z}$. The set of the quantum numbers $q$ is described by $(\kappa, l, k)$ where $\kappa=$ $0,1,2, \cdots, l=0, \pm 1, \pm 2, \cdots, k=0, \pm 2 \pi / L, \pm 4 \pi / L, \cdots$ Then, the functions $u_{q}(r)$ and $v_{q}(r)$ are expanded in terms of $\varphi_{l}^{(i)}(r)=\frac{\sqrt{2}}{J_{l+1}\left(\alpha_{l}^{(i)}\right)} J_{l}\left(\alpha_{l}^{(i)} \frac{r}{R}\right)$ as $u_{q}(r)=$ $\sum_{i} c_{q}^{(i)} \varphi_{l}^{(i)}(r)$ and $v_{q}(r)=\sum_{i} d_{q}^{(i)} \varphi_{l}^{(i)}(r)$ where $J_{l}(r)$ is the Bessel function of $l$-th order, $\alpha_{l}^{(i)}$ denotes $i$-th zero of $J_{l}$, and $R$ is a sufficiently large radius. The eigenvalue problem reduces to diagonalizing a finite matrix. Note that since the angular momentum $l$ and the wave number $k$ are both good quantum numbers, the eigenvalue equation is decomposed into each $l$ and $k$. Each blockdiagonal eigenvalue equation gives rise to the quantum number $\kappa$ along the radial direction. The iterative calculations yield a converged self-consistent solution of Eqs. (2), (3) and (4) [7].

The zero sound experiment by Andrews et al. [3] is done under the fixed total number of $\mathrm{Na}$ atoms $(\sim$ $\left.5 \times 10^{6}\right)$ and fixed axial trapping frequency $(\sim 18 \mathrm{~Hz})$ by varying the radial trapping frequency $\omega_{0}$ (typically $\sim 200 \mathrm{~Hz}$ ), giving rise to the zero sound speed as a function of the peak condensate density $n_{0}(0)$. To simulate this experiment we have performed self-consistent calculations for various $\omega_{0}$ values and various area density $n_{z}$ per unit length along the $z$-axis, which is uniform in our setting (for example, $n_{z}=2 \times 10^{4} / \mu \mathrm{m}$ ). The scattering strength is chosen as $a=2.75 \mathrm{~nm}$ [3]. The area density $n_{z}$ turns out to be one of the key parameters to fully characterize the experimental results, in particular, the zero sound velocity. The energy is scaled by $\omega_{0}$.

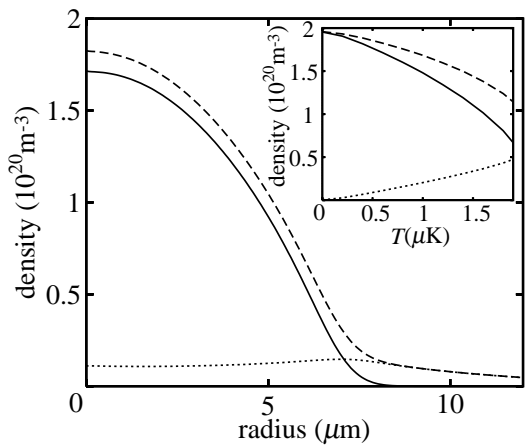

FIG. 1. Spatial profiles of the condensate $n_{0}(r)$ (full line), non-condensate $\rho(r)$ (dotted line) and total $n_{t}(r)$ (dashed line) along the radial direction. $n_{z}=2 \times 10^{4} / \mu \mathrm{m}, \omega_{0}=200 \mathrm{~Hz}$ and $T=0.6 \mu \mathrm{K}$. The inset shows the $T$-dependences of $n_{0}(0)$, $\rho(0)$ and $n_{t}(0)$.

In Fig. 11 we show a typical example of the spatial profiles of condensate $n_{0}(r)$, the non-condensate $\rho(r)$ and the total $n_{t}(r)$ along the radial direction at a finite temperature. The spatial extension of the gas is an order of $10 \mu \mathrm{m}$, which coincides with the typical radial width of a long cigar in the experiment [3]. It is also seen that the condensate concentrates at the center $r=0$, whose spatial variation is quite parabolic as predicted by TFA [5] while the non-condensate which is absent in TFA is pushed outward. The total density profile $n_{t}(r)$ deviates far from a parabolic shape. The peak condensate density $n_{0}(0)$ decreases progressively as $T$ increases as shown in inset of Fig. 1. These results are generally consistent with Hutchinson et al. 9 for a spherical case who give detailed $T$ dependence of these quantities within the same approximation.

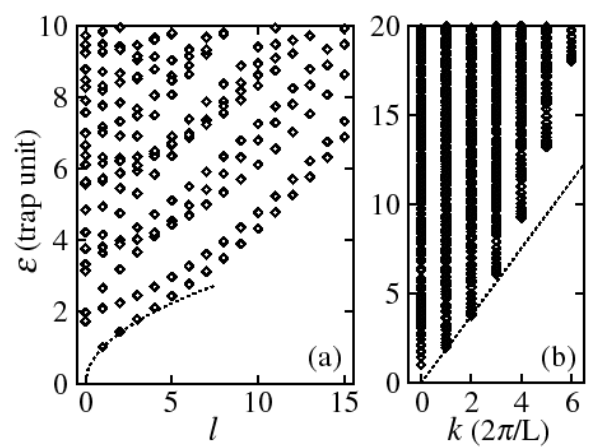

FIG. 2. Excitation spectra (a) along the angular momentum $l$ where the dotted line is the TFA prediction and (b) along the axial wave number $k$ where the dotted line is the slope of the zero sound mode. $n_{z}=2 \times 10^{4} / \mu \mathrm{m}, \omega_{0}=200 \mathrm{~Hz}$, and $T=0.6 \mu \mathrm{K}$.

The whole excitation spectra characterized by the three quantum numbers $q=(\kappa, l, k)$ are shown in Figs. 2(a) and (b). In Fig. 2(a) the energy levels are plotted as a function of the angular momentum $l$. The behavior $\varepsilon=\omega_{0} \sqrt{l}$ expected by hydrodynamic theory (or TFA) due to Stringari [8] is only approximately obeyed 
when $l$ is small in the present situation as shown by the dotted line.

The lowest three modes with $l=0,1,2$ for $\kappa=0$ are often discussed theoretically and observed experimentally on $\mathrm{Rb}$ case under a different geometrical confinement [2]. The lowest dipole mode $l=1$ coincides with $\omega_{0}$, almost independent of the interaction strength because it corresponds to a rigid oscillation of the entire trapped gas. Our result satisfies this criterion as inspected from Fig. 2(a), ensuring the accuracy of our numerical calculations. Here we only remark that the relative position of the monopole mode $\varepsilon(0,0,0)$ with $l=0$ and the quadrapole mode $\varepsilon(0,2,0)$ with $l=2$ can be reversed when the area density along the $z$-axis changes where the energy eigenvalue is denoted by $\varepsilon(\kappa, l, k)$ in terms of the above quantum numbers.

The zero sound velocity $c$ is given by the slope of the lower edge of the spectrum along the $k$ direction at $k=0$ shown in Fig. 2(b). It is clear that $c(k)$ is dispersive from a linear to quadratic curve, implying that the mode changes from the collective zero-sound to individual particle excitations toward short wave numbers (Also see Fig. (1). This crossover point corresponds to the inverse of the characteristic coherent length $\xi$ of the system.

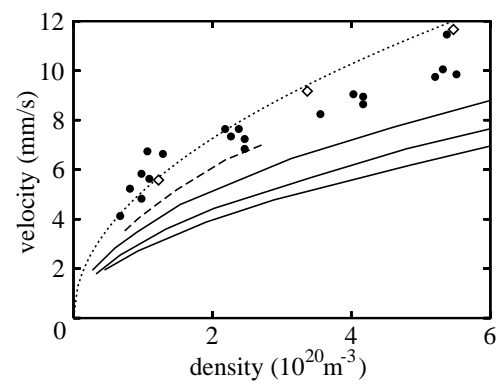

FIG. 3. Zero sound velocity $c$ against the peak condensate density $n_{0}(0)$ at low $T$. The full curves are the harmonic potential case: $n_{z}=2 \times 10^{3}, 1 \times 10^{4}$ and $2 \times 10^{4} / \mu \mathrm{m}$ from top to bottom. The topmost one nearly corresponds to Zaremba's result. The dashed line is the anharmonic case $\left(n_{z}=2 \times 10^{3} / \mu \mathrm{m}\right)$. The dots are the experimental data [3]. The diamonds are the rigid wall potential case corresponding to infinite homogeneous system (dotted line).

In Fig. 3 we plot the sound velocity $c$ as a function of the peak density $n_{0}(0)$ of the condensate. It is found that $c$ depends on the area density $n_{z}$ of the condensate along the axial direction, not only on $n_{0}(0)$. This is physically reasonable because along each curve in Fig. 3 the radial harmonic potential with $\omega_{0}$ varies, which is implicit in this figure, keeping the area density $n_{z}$ fixed. Therefore to fully characterize the system we need the additional parameter $n_{z}$. In other words, $c$ increases by increasing $\omega_{0}$ while fixing $n_{0}(0)$. These curves should be compared with that in the infinite homogeneous case. The latter is calculated by the same scheme here except that a rigid wall boundary condition is employed instead of a harmonic potential. Our calculation indeed recovers the formula $c=\sqrt{n_{0} g / m}$ when the treated system is large enough where the all relevant quantities are spatially uniform except for the boundary [7] there is no additional parameter like $n_{z}$. The velocity $c$ increases as the area density $n_{z}$ decreases as seen from Fig. 3. In the low area density limit $c$ tends to approach Zaremba's result: $c=\sqrt{n_{0} g / 2 m}$. Therefore, within the present situation of the radial harmonic confinement our velocity never exceeds Zaremba's one.

In Fig. 1 the $T$-dependence of $c$ is displayed. As $T$ decreases $c(k=0)$ decreases, signaling the loss of the rigidity of this macroscopic quantum state toward the transition temperature. In the inset of Fig. 国 the full dispersion relation $c(k)=\frac{d \varepsilon}{d k}$ of the zero sound mode is displayed. The inverse of the crossover point from a linear to quadratic curve, that is, the departure point from a constant $c(k)$ gives rise to the coherent length $\xi$ ( $\sim 0.8 \mu \mathrm{m}$ calculated for the present case). It is known that the coherent length is given by $\xi_{0}=\frac{1}{\sqrt{8 \pi a n_{0}}}$ for bulk homogeneous system.

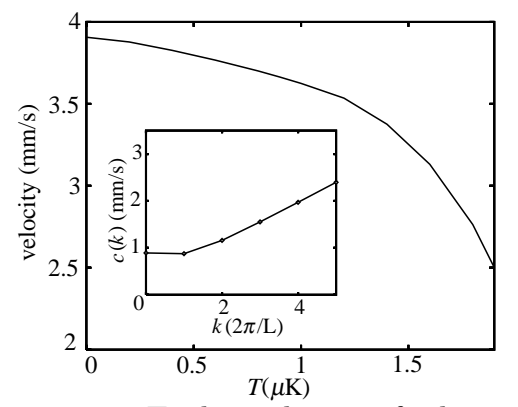

FIG. 4. $T$ dependence of the sound velocity. $n_{z}=2 \times 10^{4} / \mu \mathrm{m}$ and $\omega_{0}=200 \mathrm{~Hz}$. The inset shows the full dispersion relation $c(k)$ of the zero sound mode as a function of $k$ at $T=1.2 \mu \mathrm{K}$.

Let us now consider the experimental data for the zero sound by Andrews et al. [3]. For the reasonable area density $n_{z} \sim O\left(10^{4}\right) / \mu \mathrm{m}$ our theoretical curves are far off the experimental data as seen from Fig. 3. The experimental data are explained neither by our theoretical curves nor by the Zaremba curve based on TFA [5.6. . We argue the possible origins of this discrepancy: (1) Uncertainty of the value of the repulsive interaction $g$ or the s-wave scattering length $a$. We have assumed $a=2.75 \mathrm{~nm}$ according to [3]. As inferred from the expression $c^{2} \propto a$ for infinite system, $c$ is sensitive to the choice of the value $a$. If we choose $a=4.9 \mathrm{~nm}$ as adopted before by MIT group [10], this discrepancy may be reduced. (2) Anharmonicity of the trapping potential. The possible anharmonicity such as $\tilde{k} r^{4}$ in the trapping potential $V(r)$ may be present. An example of our result for typically $\tilde{k}=5 \times 10^{-8} \mathrm{~J} / \mathrm{m}^{4}$ is shown in Fig. 3 (dashed line), giving rise to impressive improvement of our fitting. Any anharmonicity of this type makes the condensate profile a flat-topped bulky 
shape, leading the system to more infinite homogeneous like. This results in the more bulk like sound velocity. Note that the reduction factor $\sqrt{2}$ in Zaremba's result comes from a simple geometrical reason characteristic to the parabolic shape condensate profile as mentioned before. (3) As is seen from Figs. 3 and 4 the zero sound mode is dispersive. The true zero sound measurement is only realized when exciting long wave length limiting mode. Any deviation from this limit tends to increase the estimate for the velocity as seen from inset of Fig. 4 . This might be one reason why the low density data deviates even from the infinite case, which gives the upper limit velocity. Here we point out that since the low density case has smaller aspect ratio, the sound propagation might not be purely axial mode, possibly exciting the radial mode simultaneously [11]. (4) The actual system is confined axially even though it is very long. Thus along the axial direction the density is varying spatially, this making experiment and theory for the zero sound problematic. We have performed the additional computation to see the shape and the area density in the actual experimental situations where the gas is trapped axially by a weak harmonic potential. The Gross-Pitaevskii equation mentioned before is solved under the axial trapping frequency $18 \mathrm{~Hz}$ fixed for various radial trapping frequencies $\omega_{0}$ as in the actual experiment [3]. From Fig. 5 which shows typical examples of the condensate spatial shape, it is seen that upon varying $\omega_{0}$ not only the peak density $n_{0}(0)$ but also the length and width of the condensate change, resulting in the change of the area density $n_{z}$. Generally, $n_{z}$ increases as $\omega_{0}$, or $n_{0}(0)$ increase. Thus in the actual experiment the velocity data are traversing the various curves in Fig. 3 toward the upper ones as $n_{0}(0)$ increases. We also note that according to 3 the sound velocity is position-dependent and increases near the end of the cigar. This is understandable because the "effective" density consisting of the actual density and the confining potential increases there, making $c$ larger.

In conclusion, we have calculated the whole excitation spectrum of a cylindrical symmetric Bose-Einstein gas trapped by an axial harmonic potential within the Popov approximation of the Bogoliubov framework. The zero sound compressional mode is analyzed in detail. The sound velocity and its temperature dependence are evaluated. The recent experiment by Andrews et al. [3] on $\mathrm{Na}$ gas is discussed in light of the present theory, pointing out several possible origins of the disagreement between our theory and their result on the sound velocity. It is our hope that the whole set of physical measurable quantities determined here in a self-consistent manner further serves to experimentally check the internal consistency of the Bogoliubov framework, which has been remarkably successful so far. We don't think the sound velocity an exception.

We thank D. M. Kurn, M.I.T. for helpful communication on their experiment and also T. Yabusaki and Y.
Takahashi, Kyoto Univ. for educating us regarding basics of experimental methods.

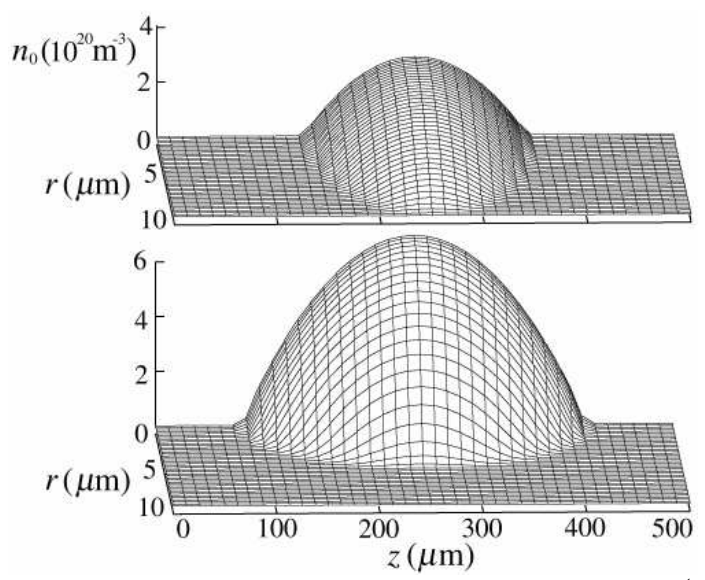

FIG. 5. Stereographic view of the condensate $n_{0}(r, z)$ at $T=0$ calculated by Gross-Pitaevskii equation at $\omega_{0}=200 \mathrm{~Hz}$ (upper) and $\omega_{0}=600 \mathrm{~Hz}$ (lower) under the axial potential fixed $(18 \mathrm{~Hz})$. The total number is $5 \times 10^{6}$. The area density $n_{z}$ at the center is seen to be $4.2 \times 10^{4} / \mu \mathrm{m}$ and $2.7 \times 10^{4} / \mu \mathrm{m}$ respectively.

* Electronic address: tomoya@mp.okayama-u.ac.jp

[1] See for reviews: A. L. Fetter, Czech. J. Phys. 46, 3063 (1997); D. S. Jin, et al., ibid. 46, 3070 (1997); N. J. van Druten, et al., ibid. 46, 3077 (1997).

[2] D. S. Jin, et al., Phys. Rev. Lett. 77, 420 (1996) on Rb; M. -O. Mewes, et al., ibid. 77, 988 (1996) on Na.

[3] M. R. Andrews, et al., Phys. Rev. Lett. 79, 553 (1997).

[4] J. Gavoret and P. Nozières, Ann. Phys. (N. Y.) 28, 349 (1964).

[5] E. Zaremba, preprint (cond-mat/9708237).

[6] G. M. Kavoulakis and C. J. Pethick, preprint condmat/9711224).

[7] See for details of numerical procedure, T. Isoshima and K. Machida, J. Phys. Soc. Jpn. 66, 3502 (1997).

[8] K. G. Singh and D. S. Rokhsar, Phys. Rev. Lett. 77, 1667 (1996); S. Stringari, ibid. 77, 2360 (1996); M. Fliesser, et al., preprint cond-mat/9706002); A. Csordás, et al., preprint (cond-mat/9705133); P. Ohberg, et al., preprint (cond-mat/9705006); D. J. Dodd, et al., preprint (condmat/9708139); D. A. W. Hutchinson and E. Zaremba, preprint (cond-mat/9709041).

[9] D. A. W. Hutchinson, et al., Phys. Rev. Lett. 78, 1842 (1997).

[10] K. B. Davis et al., Phys. Rev. Lett. 75, 3969 (1995).

[11] This is also pointed out to us by D. M. Kurn (private communication). 